\title{
A NOTE ON THE FRAME-STEWART CONJECTURE ON THE GENERALIZED TOWER OF HANOI PROBLEM
}

\author{
A. A. K. MAJUMDAR \\ Ritsumeikan Asia-Pacific University, Beppu-shi 874-8577, Japan
}

\begin{abstract}
The generalized Tower of Hanoi with $p(\geq 3)$ pegs and $n(\geq 1)$ discs, proposed by Stewart (1939) is well-known. To solve the problem, the scheme followed is : First, move the tower of the topmost $i$ (smallest, consecutive) discs (optimally) to one of the auxiliary pegs in a tower, using the $p$ pegs; next, move the remaining $n-i$ (largest) discs (optimally) to the destination peg in a tower, using the $p-1$ pegs available; and finally, transfer the discs on the auxiliary peg to the destination peg (optimally) in a tower. This is the so-called Frame-Stewart conjecture, which remains to be settled. The minimum number of moves under the scheme is denoted by $\operatorname{SF}(n, p)$. Chen and Shen (2004) have re-considered the Frame-Stewart conjecture in more detail, and claimed that $S F(n, p)$ is of the order of $2^{\left.[n(p-2) !]^{1 /(} p-2\right)}$. This paper gives a better lower bound of $S F(n, p)$, which shows that the claim of Chen et al. (2004) is not correct.
\end{abstract}

Keywords: Multi-peg Tower of Hanoi, Frame-Stewart conjecture, lower bound

\section{INTRODUCTION}

The generalized Tower of Hanoi $(\mathrm{ToH})$ problem with $p(\geq 3)$ pegs and $n(\geq 1)$ discs was proposed by Stewart (1939). Later, two solutions appeared, one by Stewart (1941) himself and the second one by Frame (1941). The problem is: Given are $p(\geq 3)$ pegs, $S, P_{1}, P_{2}, \ldots, P_{p-2}, D$. Initially, a tower of $n(\geq 1)$ discs of varying sizes, $d_{1}, d_{2}, \ldots, d_{n}$ (where $d_{1}$ is the smallest disc and $d_{n}$ is the largest one) rests on the source peg $S$, in small-on-large ordering. This is the perfect state. The objective is to shift this tower from the peg $S$ to the destination peg $D$ (using the $p-2$ auxiliary pegs $P_{1}, P_{2}, \ldots, P_{p-2}$ ), optimally (using minimum number of moves), under the "divine rule" that no disc can be placed on a smaller one.

Recently, the multi-peg $\mathrm{ToH}$ has attracted the attention of the mathematicians as well as the computer scientists, which provides a good example of recursion in computer programming.

Let $S F(n, p)$ be the minimum number of moves required to solve the problem, using the scheme below.

1 : shift (recursively) the discs $d_{1}, d_{2}, \ldots, d_{i}$ from the peg $S$ to some auxiliary peg, $P_{l}$, say, in (minimum) $S F(i, p)$ number of moves,
2: move (recursively) the remaining discs on the peg $S$ to the destination peg $D$, in minimum $S F(n-i, p-1)$ number of moves,

3 : transfer the $i$ discs from the peg $P_{l}$ to the peg $D$, again in $S F(i, p)$ moves,

where $i$ is to be determined such that the total number of moves is minimized.

Then, the DPE (dynamic programming equation) satisfied by $S F(n, p)$ is

$$
S F(n, p)=\min _{1 \leq i \leq n-1}\{2 S F(i, p)+S F(n-i, p-1)\},
$$

Unfolding the recurrence relation (1.1) further, ultimately one arrives at the situation where the topmost $n-1$ (smallest) discs on the source peg $S$ are divided into $p-2$ subtowers, each of consecutive discs, which are placed on the $p-2$ auxiliary pegs just before the largest disc $d_{n}$ is shifted from the peg $S$ to the peg $D$.

However, it remains to establish that the above scheme leading to the recurrence relation (1.1) is indeed optimal. In other words, if $M(n, p)$ is the minimum number of moves necessary to solve the p-peg ToH problem, it remains open to show that $M(n, p)=S F(n, p)$,

\footnotetext{
*Corresponding author: 〈aakmajumdar@gmail.com>
} 
which is known as the Frame-Stewart conjecture. The case when $p=3$ is almost trivial, and the optimality of the corresponding scheme has been established by Wood (1981-1982); an alternative proof appears in Hinz, Klavzar, Milutinovic and Petr (2013), under the assumption that, in the odd numbered moves, the disc $D_{1}$ is moved. In Majumdar (2012), it has been shown that, for $p=4$, the scheme followed is at least as good as any other optimal policy, and further that, for $n \geq 6$, the scheme is the only optimal policy. For $p=4$, there are other optimal schemes as well when $n=4,5$.

In an attempt to find a lower bound of $M(n, p)$, Chen and Shen (2004), following Szegedy (1999), adopted a different approach, introducing a new function $g(n, p)$, derived some results, and claimed that "This provides the strongest evidence so far to support the Frame-Stewart Conjecture".

In this paper, both the approach and results of Chen and Shen (2004) are examined. This is done in Section 2. In Section 3, the main results are given. Conclusion of the paper with some remarks is given in the final Section 4.

\section{BACKGROUND MATERIALS}

The approach and results of Szegedy (1999) are examined first since Chen and Shen (2004) are motivated by his approach. To find a lower bound of $S F(n, p)$, Szedegy (1999) starts with an arbitrary configuration $C$ of the $n$ discs on the $p$ pegs - though his analysis shows that one of the pegs must contain at least $2 m$ discs and the smallest number of discs is $n-2 p m$, where $m$ is an integer such that $0<m<\frac{n}{2 p}$. Letting $G(C)$ be the minimum number of moves necessary when it is required that each disc of $C$ is moved at least once, let

$$
g(n, p)=\min _{C} G(C),
$$

where the minimum is over all the possible configurations $C$ of the $n$ discs and $p$ pegs. Szedegy (1999) then proved that

$$
g(n, p) \geq 2 \min \{g(n-2 p m, p), g(m, p-1)\} \text {. }
$$

Further move was considered to Chen and Shen (2004). In Corollary 1 , they proved that $g(n, p)$ satisfies the following inequality:

Lemma 2.1: For $n \geq 2, p \geq 4$,

$g(n, p) \geq 2 \min \{g(n-m, p), g(m, p-1)\}$

for all $1 \leq m \leq n-1$.

Chen and Shen (2004, Lemma 4) then proved the result below which gives a lower bound of $g(n, p)$.

Lemma 2.2: For any $n \geq 1$ and $p \geq 3$,

$$
g(n, p)>2^{[n(p-2) !]^{1 /(p-2)}-p+1} \text {. }
$$

At this point, it is worth noting that both Szegedy (1999) and Chen and Shen (2004) considered $\log _{2}$ of the numbers $S F(n, p)$ and $g(n, p)$, which is completely unnecessary, since is primary interest is in the numbers $\operatorname{SF}(n, p)$ and $g(n, p)$ themselves and not their logs; moreover, $\log _{2} S F(n, p)$ and $\log _{2} g(n, p)$ do not offer any extra information or advantage.

Strictly speaking, Chen and Shen (2004) proved the following result, though in the main result (2004, Theorem 3), for some unknown reason, the factor $1-p$ (which reduces the lower bound by the multiplicative factor of $2^{p-1}$ ) has been deleted, and instead, the factor $1 \pm o(1)$ has been added. Moreover, the statement of the theorem gives a result quite different from what has actually been proved.

Proposition 2.1: For any $n \geq 1$ and $p \geq 4$,

$$
\begin{aligned}
{ }_{2}^{[n(p-2) !]^{1 /(p-2)}-p+1}<g(n, p) \leq M(n, p) \\
\leq S F(n, p)<n 2^{[n(p-2) !]^{1 /(p-2)} .} .
\end{aligned}
$$

Now examining how good are the lower and upper bounds of $S F(n, p)$, given in Proposition 2.1. For $p=4, n=18$, Proposition 2.1 is obtained that $g(18, s 4)>2^{6}$ (even disregarding the multiplicative factor $\left.2^{-3}\right)$, whereas

$\operatorname{SF}(18,4)<18 \times 2^{6}$.

Since $\operatorname{SF}(18,4)=M(18,4)=225$ (Majumdar (2012, p. 42), $2^{6}$ is a very poor lower bound and $18 \times 2^{6}$ is a too large upper bound for $\operatorname{SF}(18,4)=$ $M(18,4)$. 
Again, for $p=5$ and $n=36$, Proposition 2.1 gives $g(36,5)>2^{6}$, while

$$
\operatorname{SF}(36,5)<36 \times 2^{6} \text {. }
$$

Since $\operatorname{SF}(36,5)=M(36,5)=383$, the lower and upper bounds in Proposition 2.1 are rather poor for $p=5$ as well.

Finally, for $p=6$, and from Proposition 2.1, is obtained $g(24,6)>2^{4.5}$, and

$$
S F(24,6)<24 \times 2^{4.5} \text {. }
$$

Since $\operatorname{SF}(24,6)=M(24,6)=121$ (Majumdar 2012 the bounds obtained from Chen and Shen (2004) are indeed poor for $\operatorname{SF}(24,6)$.

It may be mentioned here that, in Proposition 2.1 , the lower bound of $\operatorname{SF}(n, p)$ is too small, while paradoxically, the upper bound of $M(n, p)$ has been made larger than $S F(n, p)$.

\section{MAIN RESULTS}

This section derives a lower bound of $\operatorname{SF}(n, p)$. For $p=4$, the following result is obtained for some integer $\mathrm{s} \geq 1$.

Lemma 3.1: Let, for some integer $\mathrm{s} \geq 1$,

$$
\frac{s(s+1)}{2}<n<\frac{(s+1)(s+2)}{2} .
$$

Then, $S F(n, 4)>s 2^{s}$.

Proof : Let

$$
n=\frac{s(s+1)}{2}+R
$$

for some integer $R$ with $1 \leq R \leq s$.

Writing the right-hand-side of the inequality (3.1) as

$$
\begin{aligned}
& (s+1)^{2}+(s+1)-2 n>0 \\
& s+1>\frac{\sqrt{8 n+1}-1}{2}>\sqrt{2 n}-\frac{1}{2},
\end{aligned}
$$

so that

$$
s>\sqrt{2 n}-\frac{3}{2} .
$$

Now,

$$
\begin{aligned}
S F(n 4) & =2^{s}\left\{\frac{s(s+1)}{2}+R-\frac{s(s-1)}{2}-1\right\}+1 \\
& =2^{s}(s+R-1)+1 \\
& >s 2^{s} \text {, if } 1 \leq R \leq s .
\end{aligned}
$$

Thus, the desired result is obtained.

The following lemma deals with the case $S F(n, p), p \geq 5$.

3.2 : Let, for $p \geq 5$,

$$
\left(\begin{array}{c}
p+s-3 \\
p-2
\end{array}\right)<n<\left(\begin{array}{c}
p+s-2 \\
p-2
\end{array}\right) \text { for some integer }
$$

$s \geq 1$, so that

$$
n=\left(\begin{array}{c}
p+s-3 \\
p-2
\end{array}\right)+R \text { for some integer } \mathrm{R}
$$

with

$$
1 \leq R \leq\left(\begin{array}{c}
p+s-3 \\
p-3
\end{array}\right)
$$

Then,

$$
S F(n, p)>(R+p-2) 2^{s}-2 p+5 .
$$

Proof : The right-hand side of the inequality (3.2) can be rewritten as follows :

$$
n<\frac{(p+s-2) !}{(p-2) ! s !}=\frac{(s+1)(s+2) \ldots(s+p-2)}{(p-2) !}
$$

so that

$$
(s+1)(s+2) \ldots(s+p-2)>n(p-2) ! .
$$

Now, by the AM-GM inequality,

$$
\begin{aligned}
& \frac{(s+1)+(s+2)+\ldots+(s+p-2)^{\frac{l}{p-2}}}{p-2} \\
& >[(s+1)(s+2) \ldots(s+p-2)]^{\frac{l}{p-2}},
\end{aligned}
$$

that is,

$$
s+\frac{p-1}{2}>[n(p-2) !]^{1 /(p-2)} .
$$

From (3.3) and (3.4),

$$
\begin{aligned}
& s>[n(p-2) !]^{1 /(p-2)}-\frac{p-1}{2} \\
& >[n(p-2) !]^{1 /(p-2)}-p+1 .
\end{aligned}
$$

The following expression of $\operatorname{SF}(n, p)$ is wellknown since the time of Frame (1941) and Stewart (1941).

$$
S F(n, p)=2^{s}\left\{n-\left(\begin{array}{c}
p+s-3 \\
p-2
\end{array}\right)\right\}+\sum_{t=0}^{s-1} 2^{t}\left(\begin{array}{c}
p+t-3 \\
p-3
\end{array}\right) .
$$

Now, for $t \geq 1$,

$$
\begin{aligned}
\left(\begin{array}{c}
p+t-3 \\
p-3
\end{array}\right) & =\frac{(t+1)(t+2) \ldots(t+p-3)}{(p-3) !} \\
& \geq \frac{(p-2) !}{(p-3) !}=p-2 .
\end{aligned}
$$


Therefore,

$$
\begin{aligned}
S F(n, p) & \geq 2^{s}\left\{n-\left(\begin{array}{c}
p+s-3 \\
p-2
\end{array}\right)\right\}+(p-2) \sum_{t=0}^{s-1} 2^{t} \\
& =R 2^{s}+(p-2)\left(2^{s}-2\right)+1,
\end{aligned}
$$

which was to be proved.

It would be instructive to see how good are the lower bounds of $S F(n, p)$, given in Lemma 3.1 and Lemma 3.2. For example, when $p=4$, $n=16, s=5$, and by Lemma 3.1,

$$
\operatorname{SF}(16,4)>5.2^{5}=160,
$$

which is almost tight, since $\operatorname{SF}(16,4)=161$. On the other hand, when $n=18, s=5$, and Lemma 3.1 gives

$$
\operatorname{SF}(18,4)>160,
$$

while $S F(18,4)=225$. The above example shows that, for the lower bound of $\operatorname{SF}(n, 4)$, the factor $R 2^{s}$ cannot be ignored. Again, when $p=5$, $n=36, s=5, R=1$, and from Lemma 3.2,

$$
\operatorname{SF}(36,5)>123 .
$$

Lemma 3.1 shows that, $\operatorname{SF}(n, 4)$ is of the order $\left(\sqrt{2 n}+R-\frac{5}{2}\right) 2^{\sqrt{2 n}-\frac{3}{2}}$; also by virtue of Lemma 3.2, $S F(n, p)$ is of the order $(R+p-2) 2^{[n(p-2) !]^{\frac{1}{1-p}}-p+1}$ for $p \geq 5$, where in the latter case, the lower bound is rather a very conservative estimate.

With the improved lower bound of $\operatorname{SF}(n, p)$, the problem needs to be considered.

\section{CONCLUSION}

Chen and Shen (2004, Theorem 3), actually proved that the newly defined function $g(n, p)$ is of the order $2^{[n(p-2) !]^{\frac{1}{p-2}}-p+1}$, and concluded, without further closer study, that $S F(n, p)$ is of the same order. As has been proved in Lemma 3.1 and Lemma 3.2, SF $(n, p)$ is not of the same order as $g(n, p)$. Moreover, since $S F(n$, $p$ ) itself is an upper bound for $M(n, p)$, and since an explicit form of $S F(n, p)$ is already available, it is unnecessary to look for an upper bound for
$M(n, p)$. As has already been pointed out, the upper bound of $S F(n, p)$ (and $M(n, p)$ as well), given in Proposition 2.1 is rather very crude.

Surprisingly, the same erroneous lower bound for $g(n, p)$ has been quoted in Hinz, Klavzar, Milutinovic and Petr (2013) as

$$
2^{(1+o(1))[n(p-2) !]^{\frac{1}{p-2}},}
$$

adding the term $1+o(1)$ in the exponent, apparently without realizing that actually only the left-hand side of the inequality in Proposition 2.1 has been established. It may be mentioned here that, if the above result is correct, it would be $g(36,5)>2^{12}$.

In the Tower of Hanoi problem, one has to start with the tower in perfect state on the source peg $S$, and the approach of Szedegy (1999) and Chen and Shen (2004) does not indicate how to dismantle this tower in order to move it to the destination peg $D$. It is thus unnecessary to introduce an artificial function $g(n, p)$ in connection with the Frame-Stewart conjecture, particularly since it does not give any further insight into the problem. and the only property known about the function $g(n, p)$ is the inequality (2.2), together with the inequality $g(n, p) \leq M(n, p)$.

It is thus seen that the criterion that tracfind tracking only on the number of moves ensuring (at least) one movement of each disc would not help us at all in resolving the Frame-Stewart conjecture. Moreover, under that criterion, one would end up with a configuration that is not perfect, though the problem is to transfer the tower of $n$ discs in perfect state on the source peg to the perfect state on the destination peg (in minimum number of moves). Moreover, it is not known how $g(n, p)$ is (functionally) related to $S F(n, p)$. It is also not known how the newly found lower bound of $\operatorname{SF}(n, p)$ is related to $M(n$, $p$ ) or to $g(n, p)$. From Proposition 2.1, it could not be claimed that $S F(n, p)$ and $M(n, p)$ are of the same order.

Thus, the Frame-Stewart conjecture still remains open. However, it is conjectured that, for 
sufficiently large values of $n$, the scheme leading to the recurrence relation (1.1) is not only optimal, it is the only optimal policy to solve the p-peg ToH. This is based on the fact that, for the generalized p-peg ToH problem with $(p \geq 4)$ pegs and $n$ discs, the scheme is the only one to solve the problem optimally when

$$
n=\left(\begin{array}{c}
p+s-3 \\
p-2
\end{array}\right), s \geq 1 \text {. }
$$

The same point of view has been shared by Hinz, Klavzar, Milutinovic and Petr (2013, Conjecture 5.41). It may be recalled here that, the solution of the classical ToH problem (with 3 pegs and $\mathrm{n}$ discs) is unique, when $p=4$, the solution is still unique if $n=3$, and unique again when $n=6$, and for $n=4,5$, there are solutions in addition to the pms (presumed minimum solution) determined by (1.1). This, in turn, implies that the ToH problem with $p(\geq 5)$ pegs has non-pms solutions for sufficiently large values of $n$. It may also be recalled that, for $p \geq 5, F S(n, p)$ is uniquely attained at the point $k=\left(\begin{array}{c}p+s-4 \\ p-2\end{array}\right)$ when $n=\left(\begin{array}{c}p+s-3 \\ p-2\end{array}\right), \quad$ and simultaneously, the function $F S$

$\left(\left(\begin{array}{c}p+s-3 \\ p-2\end{array}\right)-\left(\begin{array}{c}p+s-3 \\ p-2\end{array}\right), p-1\right)$ is uniquely attained. Thus, for example, $F S(10,5)$ is attained at the unique point $k=K=4$, because each of the two functions $F S(K, 5)=F S(4,5)$ and $F S(10-N, 4)=F S(6,4)$, is attained at a unique point. For the analysis of the generalized p-peg ToH problem, this fact should be kept in mind.

\section{ACKNOWLEDGEMENT}

Part of the research was done during the ADL (Academic Development Leave) period. The author acknowledges with thanks Ritsumeikan Asia-Pacific University, Japan, for granting the ADL.

\section{REFERENCES}

Chen, X. and J. Shen. 2004. On the FrameStewart Conjecture about the Towers of Hanoi. SIAM J. Comput. 33(3): 584-589.

Frame, J.S. 1941. Solution to AMM Problem 3918. American Mathematical Monthly. 48: 216-219.

Hinz, A. M., S. Klavzar, U. Milutinovic and C. Petre. 2013. The Tower of Hanoi - Myths and Maths. Springer, NY.

Majumdar, A. A. K. 2012. The Classical Tower of Hanoi Problem and Its Generalizations Vol. 1 : Multi-Peg Generalization. Lambert Academic Publishing.

Stewart, B. M. 1939. Problem 3918. American Mathematical Monthly, 46: 363.

Stewart, B. M. 1941. Solution to AMM Problem 3918, American Mathematical Monthly, 48: $216-219$.

Szegedy, M. 1999. In How Many Steps the p Peg Version of the Towers of Hanoi Game Can be Solved. Lecture Notes in Computer Science, 1563: 356 - 361.

Wood, D. 1981-1982. The Towers of Brahma and Hanoi Revisited, Journal of Recreational Mathematics, 14(1) : 17-24.

(Received revised manuscript on 02 April 2019) 